\title{
Fermentación cecal in vitro de cerdos Pelón Mexicano y Cuino Mexicano suplementados con cromo
}

\section{In vitro caecal fermentation in Cuino Mexican and Hairless Mexican pigs supplemented with chromium}

\author{
Ignacio Arturo Domínguez-Vara, Adriana Emilia Gómez-Galeana, Nazario Pescador-Salas, Manuel \\ González-Ronquillo *
}

\author{
Departamento de Nutrición Animal, Facultad de Medicina Veterinaria y Zootecnia, \\ Universidad Autónoma del Estado de México, Instituto Literario 100, CP. 50000 . Toluca, \\ Estado de México, México. \\ *Autor de correspondencia: mrg@uaemex.mx
}

Artículo científico recibido: 30 de mayo de 2016 aceptado: 17 de noviembre de 2016

RESUMEN. Los cerdos Pelón Mexicano (CPM) y Cuino Mexicano (CCM) son nativos de México. El cromo ( $\mathrm{Cr}$ ) es un nutriente esencial en el metabolismo de carbohidratos, lípidos y aminoácidos, pero su efecto en cerdos a nivel de la fermentación cecal se desconoce. El objetivo fue evaluar la fermentación cecal in vitro y el nitrógeno (N) digestible en el intestino del CPM y CCM, mediante la adición de $\mathrm{Cr}$ en la dieta. Se sacrificaron 48 cerdas con peso vivo de $104 \pm 0.5 \mathrm{~kg}, 24$ por cada genotipo, de las cuales se colectó contenido cecal e ileal. Las muestras de contenido ileal se

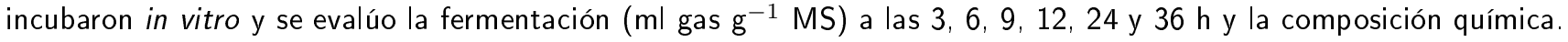
El diseño experimental fue completamente al azar con arreglo factorial $2 \times 2$. No hubo diferencias entre genotipos ( $\mathrm{P}>0.1$ ), la dieta sin $\mathrm{Cr}$ mostró mayor fermentación $\left(\mathrm{p}<0.001\right.$ ), la producción de gas acumulada ( $\mathrm{ml}$ gas $\left.\mathrm{g}^{-1} \mathrm{MS}\right)$ no mostro diferencias $(\mathrm{P}>0.1)$ por efecto del genotipo o dieta. Hubo mayor contenido de $\mathrm{N}$ total y $\mathrm{N}-\mathrm{NH}_{3}$ en íleon para el genotipo CCM $(p<0.05)$. No hubo diferencia $(\mathrm{P}>0.05)$ en el contenido de $\mathrm{N}$-total, $\mathrm{N}^{-\mathrm{NH}_{3}}$ y $\mathrm{N}$ aminoacidico a nivel cecal. Se concluye que los CCM mostraron un mayor contenido de N-Total y N-NH en íleon que los CPM; el $\mathrm{Cr}$ dietario no influyó en el contenido de nitrógeno en intestinos delgado y grueso, ni en la fermentación cecal microbiana de los cerdos.

Palabras clave: Cerdos nativos, producción de gas in vitro, proteína digestible, cromo

ABSTRACT. The Mexican hairless (MHP) and Mexican cuino (MCP) pigs are native to Mexico. Chromium (Cr) is an essential nutrient in the metabolism of carbohydrates, lipids and amino acids, but its effect on pigs at the level of cecal fermentation is unknown. The objective was to evaluate the in vitro cecal fermentation and digestible nitrogen $(\mathrm{N})$ in the intestine of the MHP and MCP, by adding $\mathrm{Cr}$ in the diet. Forty-eight sows with a live weight of $104 \pm 0.5 \mathrm{~kg}$ were slaughtered, 24 for each genotype, from which cecal and ileal contents were collected. The ileal content samples were

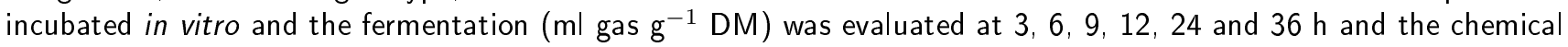
composition. The experimental design was completely randomized with a $2 \times 2$ factorial arrangement. There were no differences between genotypes $(P>0.1)$, the diet without $C r$ showed higher fermentation $(P<0.001)$, and cumulative gas production ( $\mathrm{ml}$ gas $\left.\mathrm{g}^{-1} \mathrm{DM}\right)$ showed no differences $(\mathrm{P}>0.1)$ due to genotype or diet effect. There were higher total $\mathrm{N}$ and $\mathrm{N}-\mathrm{NH}_{3}$ contents in ileum for the MCP genotype $(\mathrm{P}<0.05)$. There were no differences $(\mathrm{P}>0.05)$ in total $\mathrm{N}, \mathrm{N}-\mathrm{NH}_{3}$ and $\mathrm{N}$ amino acid contents at cecal level. It was concluded that MCP showed a higher content of total $\mathrm{N}$ and $\mathrm{N}-\mathrm{NH}_{3}$ in ileum than MHP; dietary $\mathrm{Cr}$ did not influence the nitrogen content in the small and large intestines or in the microbial cecal fermentation of the pigs.

Key words: Native pigs, in vitro gas production, digestible protein, chromium.

\section{INTRODUCCIÓN}

El cerdo Pelón Mexicano (CPM) ha sido desplazado por la introducción de otras razas de cerdos mejorados; lo que ha disminuido la población porcina criolla (Sierra-Vásquez 2000). Actualmente, cerca del $30 \%$ de los recursos genéticos de animales 
domésticos del mundo, están catalogados con un alto riesgo de extinción, entre ellos el CPM (FAO 2007). México cuenta con algunos genotipos porcinos autóctonos, los cuales se conocen como el Cerdo Pelón Mexicano (CPM) y Cerdo Cuino Mexicano (CCM), ambos genotipos provienen del Cerdo Nativo Mexicano (Lemus et al. 2003).

El cromo $(\mathrm{Cr})$ es un micromineral esencial involucrado en el metabolismo de los carbohidratos, lípidos y aminoácidos (García-Castillo et al. 2004), es necesario en la síntesis de proteínas y en el crecimiento muscular (Mertz 1993). Se ha observado que su suministro en la dieta mejora el rendimiento animal y aumenta el metabolismo energético (Kitchalong et al. 1995), principalmente de carbohidratos (glucosa). El Cr es un componente activo del factor de tolerancia a la glucosa (FTG), molécula cuya acción potencializa la acción de la insulina en la célula (Amoikon et al. 1995). Es un micromineral que actúa en el metabolismo de los nutrientes a nivel celular, promoviendo el crecimiento tisular; al respecto Kornegay et al. (1997) detectaron que el $\mathrm{Cr}$ puede influir en el crecimiento y en las características de la canal, aumentando la eficiencia de uso del nitrógeno de la dieta. Lo anterior está respaldado por Lindeman et al. (1995) quienes calcularon el nitrógeno retenido en canales de cerdos suplementados con $\mathrm{Cr}$, indicando un aumento significativo del nitrógeno retenido en la canal, así como un ahorro del nitrógeno excretado en heces. $\mathrm{El} \mathrm{Cr}$ al ser parte de la estructura del FTG, actúa en el metabolismo de los nutrientes, azucares, lípidos y aminoácidos (Arvizu et al. 2011), pero poco se ha estudiado su función en la fermentación a nivel retículo ruminal en rumiantes o en la fermentación cecal del cerdo.

En los cerdos, los carbohidratos y proteínas, así como el material digestivo endógeno y las células bacterianas, no digeridos en el intestino delgado, pasan por la válvula íleo-cecal y en el intestino grueso son fermentados por la microflora (Högberg et al. 2004). Los carbohidratos no digeribles son la principal fuente de energía para las bacterias presentes en el intestino grueso (Bindelle et al. 2007), de esta forma, su fermentación pro- duce un cambio de la excreción de nitrógeno ureico en orina y de la proteína en las heces (Zervas y Zijlstra 2002). La fermentación microbiana que ocurre en el intestino grueso del cerdo, principalmente en el ciego, produce ácidos grasos de cadena corta (Suarez-Belloch et al. 2013), los cuales aportan de 10 a $30 \%$ de sus necesidades de energía para mantenimiento (Hume 1997); también se producen $\mathrm{CO}_{2}, \mathrm{CH}_{4}$ y N-NH $\mathrm{NH}_{3}$ (Jezierny et al. 2007). En los cerdos, la fermentación microbiana cecal puede estimarse mediante la técnica de producción de gas in vitro (Fondevila et al. 2002, Domínguez-Vara et al. 2010). Por lo anterior el objetivo del presente estudio fue evaluar el efecto del cromo en la dieta de cerdos Pelón Mexicano y Cuino Mexicano en finalización, sobre el contenido de nitrógeno ileal y cecal, en los parámetros de fermentación microbiana cecal.

\section{MATERIALES Y MÉTODOS}

\section{Animales y dietas}

Se utilizaron 48 cerdas, 24 del genotipo Cerdo Pelón Mexicano y 24 del genotipo Cerdo Cuino Mexicano, alojadas en corraletas con tres cerdas cada una, las cuales se alimentaron ad libitum con una dieta estándar para la etapa de finalización (64 d) y agua ad libitum. La dieta se elaboró con 76.8 $\%$ de maíz, $7.9 \%$ de canola, $7.0 \%$ de pasta de soya, $5.0 \%$ de salvado de trigo, $2.5 \%$ de vitaminas y minerales, $0.6 \%$ de $\mathrm{CaCO}_{3}$ y $0.2 \%$ de $\mathrm{NaCl}$; con una concentración energética de $2.58 \mathrm{Mcal}$ de EM $\mathrm{kg}^{-1} \mathrm{MS}, 15.6 \%$ de proteína cruda (PC), $3.78 \%$ de extracto etéreo (EE), $0.88 \%$ de Calcio (Ca) y $0.69 \%$ de fósforo $(\mathrm{P})$. Un grupo de 12 cerdas de cada genotipo fue suplementado con $2.5 \mathrm{~g}^{\text {cerdo }^{-1}}$ día $^{-1}$ de la premezcla Cr-L Metionina (1000 ppm de $\mathrm{Cr}$ ) y el otro grupo se usó como control. Las dietas se muestrearon cada semana y se hizo un pool para su posterior análisis. Cuando las cerdas alcanzaron un peso vivo de $104 \pm 0.5 \mathrm{~kg}$, tras un ayuno de $10 \mathrm{~h}$, con acceso a agua, se sacrificaron en el rastro Tipo Inspección Federal ubicado en Cuautitlán Izcalli, Estado de México. Se realizaron cuatro periodos de sacrificio, 12 animales por periodo, seis 
de cada genotipo, tres con suplemento de $\mathrm{Cr}$ y tres $\sin \mathrm{Cr}$. Los cerdos siempre se encontraban en buen estado de salud. El estudio se aprobó por el Comité de Ética de la Facultad de Medicina Veterinaria y Zootecnia de la Universidad Autónoma del Estado de México.

\section{Toma de muestras}

Sacrificadas las cerdas y extraídos los órganos digestivos abdominales, se colectaron $100 \mathrm{~g}$ de contenido ileal y $250 \mathrm{~g}$ de contenido cecal de cada cerda; posteriormente, se mezclaron y homogenizaron los contenidos ileales (sustrato), y cecales (inóculo), de tres cerdas del mismo tratamiento. Las muestras se conservaron en refrigeración a $4{ }^{\circ} \mathrm{C}$ por $3 \mathrm{~h}$ y se transportaron al laboratorio de Bromatología del Departamento de Nutrición Animal de la Facultad de Medicina Veterinaria y Zootecnia de la UAEM, Campus Universitario el Cerrillo, Toluca, México, para su procesamiento. El contenido ileal se mantuvo por cerdo, genotipo y tratamiento en refrigeración, este último se deshidrató a $60{ }^{\circ} \mathrm{C}$ por 48h. Cada mezcla de contenido cecal se utilizó como inoculo para la incubación in vitro de cada mezcla de contenido ileal.

\section{Producción de gas in vitro}

La producción de gas in vitro se determinó por el método propuesto por Theodorou et al. (1994). Se utilizaron por cada sustrato, con y $\sin \mathrm{Cr}$, y por cada genotipo, CPM y CCM, tres frascos con capacidad de $125 \mathrm{~mL}$ para cada muestra; en el que se pusieron $800 \mathrm{mg}$ de MS del contenido ileal de cada cerda y se adicionaron $90 \mathrm{~mL}$ de solución buffer, se aplicó gas $\mathrm{CO}_{2}$, y se mezcló con $700 \mathrm{~mL}$ de solución buffer y $300 \mathrm{~g}$ de contenido cecal (Fondevila et al. 2002).

La mezcla de contenido cecal de cada sustrato y genotipo se filtró en cuatro capas de gasa $y$, posteriormente, en lana de vidrio, después se introdujeron $10 \mathrm{~mL}$ del inóculo cecal en cada frasco. Los frascos se pusieron en un baño de incubación con agua a $39{ }^{\circ} \mathrm{C}$, para iniciar el registro de las lecturas de producción de gas a las 3, 6, 9, 12, 18, 24 y $36 \mathrm{~h}$, con un transductor de presión (DELTA OHM,
Manometer 8804). Para ajustar la contribución potencial de otros extractos solubles en la producción de gas y corregir las lecturas de los sustratos en la fermentación cecal, se utilizaron tres frascos sin sustrato como blancos, y tres frascos con sustrato de paja de avena como estándares. La producción de gas se determinó con el valor promedio de gas producido por hora.

Para la suma aditiva de la producción de gas se utilizó la ecuación propuesta por France et al. (1993) $\mathrm{PG}=\mathrm{a}\left\{1-e-b^{(t-T)}-c^{(\sqrt{t}-\sqrt{T})}\right\}$, donde: $\mathrm{PG}$, producción de gas acumulada ( $\mathrm{mL}$ gas/g MS); a es la asíntota de la curva (producción de gas total, $\mathrm{mL}) ; \mathrm{b}\left(\mathrm{h}^{-1}\right)$ y $\mathrm{c}\left(\mathrm{h}^{-1 / 2}\right)$ son constantes de producción de gas y $\mathrm{T}(\mathrm{h})$ es el tiempo de retardo antes de que la fermentación inicie, los datos se analizaron mediante el programa Grafit (1992). También se determinó la producción de gas por hora (Domínguez-Vara et al. 2010).

\section{Composición química}

Las muestras de las dietas alimentadas a las cerdas se molieron en un molino Willey a tamaño de $1 \mathrm{~mm}$, y se analizaron por duplicado para determinar el contenido de $\mathrm{MS}$ a $60{ }^{\circ} \mathrm{C}$ por $48 \mathrm{~h}$, $\mathrm{N}$ por el método $\mathrm{N}-\mathrm{Kjeldahl}$ utilizando cobre como catalizador, extracto etéreo por Soxhlet (AOAC 1990) y el contenido de FND (Van Soest et al. 1991) con alfa amilasa sin corrección por cenizas. En las muestras de contenido ileal y cecal se determinó la MS, el $\mathrm{N}$ total, el $\mathrm{N}-\mathrm{NH}_{3}$ y el $\mathrm{N}$ aminoacidico ( $\mathrm{N}$ aa) (Firkins et al. 1992), contenido de FND (Van Soest et al. 1991) con alfa amilasa sin corrección por cenizas. Para la determinación del cromo, se solubilizo el $\mathrm{Cr}$ en las dietas, el contenido ileal y cecal por digestión acida $\left(\mathrm{HNO}_{3}\right.$ y $\left.\mathrm{HClO}_{4}\right)$ en una parrilla (Allan 1971) y se cuantifico con espectrofotometría de absorción atómica.

\section{Análisis estadístico}

La información de la incubación in vitro se analizó bajo un diseño completamente al azar con arreglo factorial $2 \times 2$ con dos genotipos y dos sustratos (con y $\sin \mathrm{Cr}$ ), y tres repeticiones (periodos de incubación) con el programa SAS 
(SAS 1999). El modelo utilizado fue: $Y_{i j k}=$ $\mu+A_{i}+B_{j}+A B_{i j}+\varepsilon_{i j k}$. Donde: $Y_{i j k}=$ Variable de respuesta, $\mu=$ Media general, $\mathrm{A}_{i}=$ Efecto de genotipo, $\mathrm{B}_{j}=$ Efecto del sustrato con $\mathrm{Cr}$ y $\sin$ $\mathrm{Cr}, \mathrm{AB}_{i j}=$ Efecto de la interacción genotipo con sustrato, $\varepsilon_{i j k}=$ Error experimental. Los promedios de cada variable $(p<0.05)$ se compararon con la prueba de Tukey (Steel et al. 1997).

\section{RESULTADOS}

En la Tabla 1 se muestra la producción de gas in vitro mediante la fermentación cecal de cerdos CPM y CCM con y $\sin \mathrm{Cr}$; la producción total de gas no mostró diferencias entre genotipos $(P=0.613)$, pero fue mayor $(p<0.001)$ para el sustrato sin $\mathrm{Cr}$; con respecto al efecto de la interacción, el valor medio fue mayor $(p<0.002)$ para los cerdos CPM $\sin \mathrm{Cr}$. La fracción b mostró diferencias por efecto de la interacción del genotipo con el $\mathrm{Cr}$, siendo superior $(\mathrm{p}<0.012)$ para los cerdos $\mathrm{CCM}$ con $\mathrm{Cr}$; la fracción $c$, no mostró diferencias entre genotipos $(P=0.666)$, ni efecto de la interacción del genotipo con el $\mathrm{Cr}(\mathrm{P}=0.440)$. El efecto del $\mathrm{Cr}$ fue mayor ( $\mathrm{p}$ $<0.033$ ) en los cerdos que no fueron suplementados. El tiempo de retardo fue superior $(p<0.01)$ en los cerdos CCM, pero sin diferencias por efecto del $\mathrm{Cr}(\mathrm{P}=0.772)$ y la interacción de genotipo con $\mathrm{Cr}(\mathrm{P}=0.151)$. La producción de gas acumulada ( $\mathrm{ml}$ gas $\mathrm{h}^{-1}$ ) entre genotipos, mostró una tendencia de cambio $(p<0.08)$ a las $3,6,24$ y 36 h, con valores superiores para los cerdos CPM. El efecto debido al $\mathrm{Cr}$ en la dieta sobre la producción de gas acumulada por hora no fue diferente $(\mathrm{P}>0.1)$; mientras que la interacción del genotipo con el $\mathrm{Cr}$, solo mostró diferencia $(\mathrm{p}<0.03)$ a las $3 \mathrm{~h}$.

En la Figura 1 se observa la producción de gas por hora $\left(\mathrm{ml}\right.$ gas $\mathrm{h}^{-1}$ ) para ambos genotipos de cerdos; en las primeras horas de fermentación no hubo diferencias $(P>0.05)$, mientras que a las 18 , 24 y $36 \mathrm{~h}$ se tuvieron diferencias $(p<0.05)$ en la producción de gas en los cerdos CPM con respecto a los cerdos CCM. En la Figura 2 se muestra el efecto del $\mathrm{Cr}$, se observó un aumento $(\mathrm{p}<0.05)$ en la producción de gas para la dieta $\sin \mathrm{Cr}$ a las 18 y 24 $h$, pero sin diferencias $(P>0.05)$ con el resto de las horas de incubación.

En la Tabla 2 se aprecian las concentraciones de $\mathrm{Cr}$, FND, N-total, $\mathrm{N}-\mathrm{NH}_{3}$ y $\mathrm{N}$-aa en el contenido ileal y cecal de los cerdos; el nivel de $\mathrm{Cr}$ en el contenido cecal fue mayor $(p<0.01)$ en los cerdos que consumieron $0.25 \mathrm{ppm}$ de $\mathrm{Cr}$ en la dieta, pero no hubo diferencias $(P=0.752)$ en el contenido ileal de $\mathrm{Cr}$ entre genotipos $(\mathrm{P}=0.373)$ y sustratos $(P=0.949)$. Pero se observó una tendencia a cambiar $(\mathrm{P}=0.092)$ por efecto de la interacción del genotipo con el $\mathrm{Cr}$, siendo superior en los cerdos CPM sin $\mathrm{Cr}$. Para el contenido cecal de $\mathrm{Cr}$, no se observaron diferencias $(P=0.752)$ entre genotipos; pero el $\mathrm{Cr}$ en el contenido cecal fue $75 \%$ mayor en los cerdos que consumieron la dieta con $\mathrm{Cr}(\mathrm{p}<$ 0.01).

La concentración de $\mathrm{N}$-total a nivel ileal fue superior $(p<0.004)$ para los cerdos CCM con respecto a los $C P M$, pero no se encontraron diferencias por efecto del $\mathrm{Cr}(\mathrm{P}=0.178)$, ni de la interacción $(\mathrm{P}=0.766)$ del genotipo con el $\mathrm{Cr}$. $\mathrm{El}$ contenido de $\mathrm{N}-\mathrm{NH}_{3}$ fue superior $(p<0.009)$ en los cerdos CCM con respecto a los cerdos CPM, pero no hubo efecto del $\mathrm{Cr}(\mathrm{P}=0.385)$, ni de la interacción $(\mathrm{P}=0.447)$. La concentración de $\mathrm{N}$-aa no mostró diferencias entre genotipos $(P=0.163)$, ni por efecto del $\mathrm{Cr}(\mathrm{P}=0.412)$, igualmente la concentración de $\mathrm{N}$ cecal no tuvo diferencias $(\mathrm{P}>0.10)$ por efecto del genotipo ni del $\mathrm{Cr}$ en la dieta.

\section{DISCUSIÓN}

La PG acumulada en la fermentación cecal no fue diferente entre los genotipos de cerdos, solo se observaron diferencias en las últimas horas en la fermentación cecal de los cerdos CPM con respecto a los cerdos CCM (Figura 1). Pero este efecto se pierde al combinar el contenido cecal de ambos genotipos con $\mathrm{Cr}$ y $\sin \mathrm{Cr}$, excepto a las $24 \mathrm{~h}$ (Figura 2), donde la dieta sin $\mathrm{Cr}$ fue superior ( $\mathrm{p}<$ $0.05)$. Estos resultados difieren de lo reportado por Domínguez-Vara et al. (2010), quienes indican que el CPM posee una mayor capacidad fermentativa cecal que el cerdo CCM, ya que es probable que los mi- 
Tabla 1. Producción de gas in vitro de cerdos Pelón Mexicano (CPM) y Cuino Mexicano (CCM) suplementados con y sin cromo en la dieta, con contenido ileal como sustrato.

\begin{tabular}{|c|c|c|c|c|c|c|c|c|}
\hline \multirow[t]{2}{*}{ Variable } & \multicolumn{2}{|c|}{ Genotipo } & \multicolumn{2}{|c|}{ Sustrato (ileal) } & \multirow[b]{2}{*}{ EEM } & \multicolumn{3}{|c|}{$\mathrm{p}<$} \\
\hline & CPM & CCM & $\mathrm{Cr}$ & $\sin C r$ & & Genotipo & Sustrato & GxS \\
\hline $\mathrm{A}^{\dagger}$ & 131.35 & 126.67 & $111.00^{e}$ & $147.02^{d}$ & 6.44 & 0.613 & 0.001 & 0.002 \\
\hline B & 0.0903 & 0.1346 & 0.1328 & 0.0921 & 0.02 & 0.116 & 0.147 & 0.012 \\
\hline C & -0.2254 & -0.1888 & $-0.3030^{e}$ & $-0.1112^{d}$ & 0.06 & 0.666 & 0.033 & 0.440 \\
\hline Lag time & $1.14^{e}$ & $1.91^{d}$ & 1.48 & 1.57 & 0.20 & 0.010 & 0.772 & 0.151 \\
\hline \multicolumn{9}{|c|}{ Producción de gas ( $\mathrm{ml}$ gas $\mathrm{g}^{-1} \mathrm{MS}$ ) } \\
\hline $3 \mathrm{~h}$ & 10.08 & 7.76 & 10.30 & 7.20 & 0.78 & 0.080 & 0.468 & 0.031 \\
\hline $6 \mathrm{~h}$ & 33.84 & 31.72 & 36.86 & 28.04 & 2.08 & 0.067 & 0.269 & 0.791 \\
\hline $9 \mathrm{~h}$ & 48.09 & 49.04 & 54.24 & 42.08 & 3.23 & 0.132 & 0.289 & 0.833 \\
\hline $12 \mathrm{~h}$ & 63.37 & 64.10 & 70.13 & 56.56 & 4.17 & 0.199 & 0.341 & 0.683 \\
\hline $18 \mathrm{~h}$ & 72.27 & 70.89 & 77.48 & 64.82 & 4.47 & 0.116 & 0.476 & 0.386 \\
\hline $24 \mathrm{~h}$ & 77.32 & 75.65 & 81.63 & 70.57 & 4.71 & 0.097 & 0.636 & 0.109 \\
\hline $36 \mathrm{~h}$ & 80.86 & 78.97 & 84.79 & 74.29 & 4.88 & 0.088 & 0.705 & 0.109 \\
\hline
\end{tabular}

Letras distintas en la misma hilera son estadísticamente diferentes $(\mathrm{p}<0.05)$. G: efecto del genotipo (CPM vs CCM), S: efecto del sustrato (Contenido ileal con $\mathrm{Cr}$ y sCr). †'Ecuación de France et al. (1993), A: asíntota de la curva (producción de gas total, $\mathrm{mL}$ ), b: $\left(\mathrm{h}^{-1}\right)$ y c: $\left(\mathrm{h}^{-1 / 2}\right)$ son constantes de producción de gas y Lag time $(\mathrm{h})$ es el tiempo de retardo antes de que la fermentación inicie, EEM: error estándar de la media.

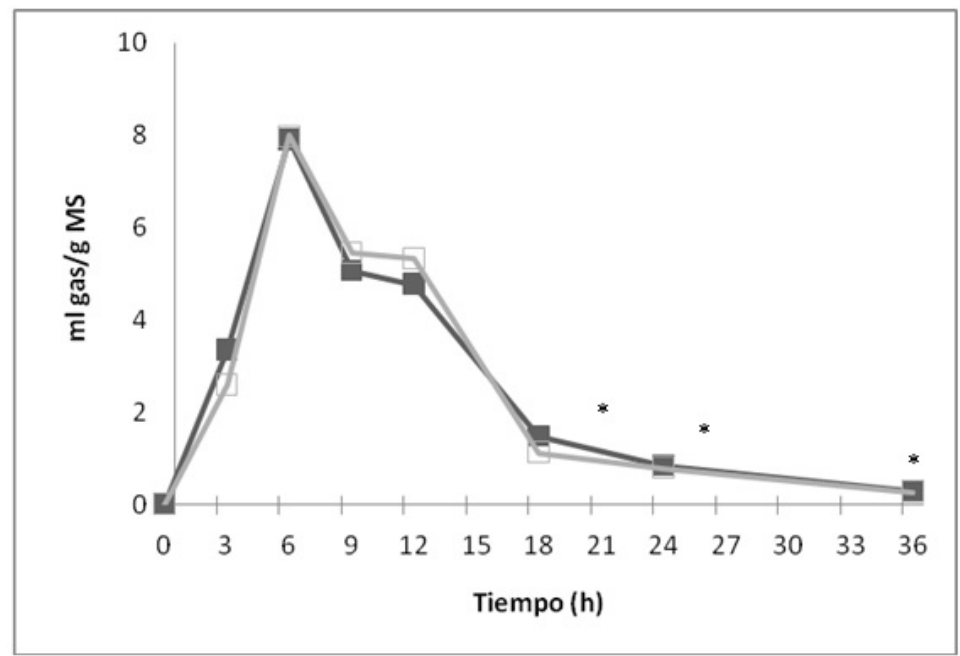

Figura 1. Producción de gas in vitro $\left(\mathrm{ml}\right.$ gas $\left.\mathrm{g} \mathrm{MS}^{-1}\right)$ por hora del contenido ileal de cerdos Pelón Mexicano ( $\mathbf{\square})$ y Cuino Mexicano $(\square)(*, \mathrm{p}<0.05)$.

croorganismos presentes en el ciego del CPM tengan mayor actividad enzimática con respecto al cerdo CCM. Una de las funciones del intestino grueso es la conservación de la microflora (Cummings 1983); actualmente se acepta que a través de la fermentación cecal se aporta energía y nutrientes por la simbiosis de la micro flora del tracto gastrointestinal (Williams et al. 2001). Los principales carbohidratos dietéticos para el cerdo son el almidón, los azúcares y la fibra (Durmic et al. 2000, Högberg et al. 2004); esta última, al ser potencialmente degradable en el ciego por la acción bacteriana, permite la producción de ácidos grasos volátiles como fuente de energía.

La PG a nivel cecal ocurre, por la presencia de fibra (De Schrijver et al. 1999), ya que llega poco almidón fermentable a este órgano, y por la acción de los microorganismos presentes (Regmi et al. 2011). Al respecto Fondevila et al. (2002) indican que la $P G$ in vitro con inóculo cecal de cerdos Landrace fue mayor en las primeras $8 \mathrm{~h}$; lo que coin- 


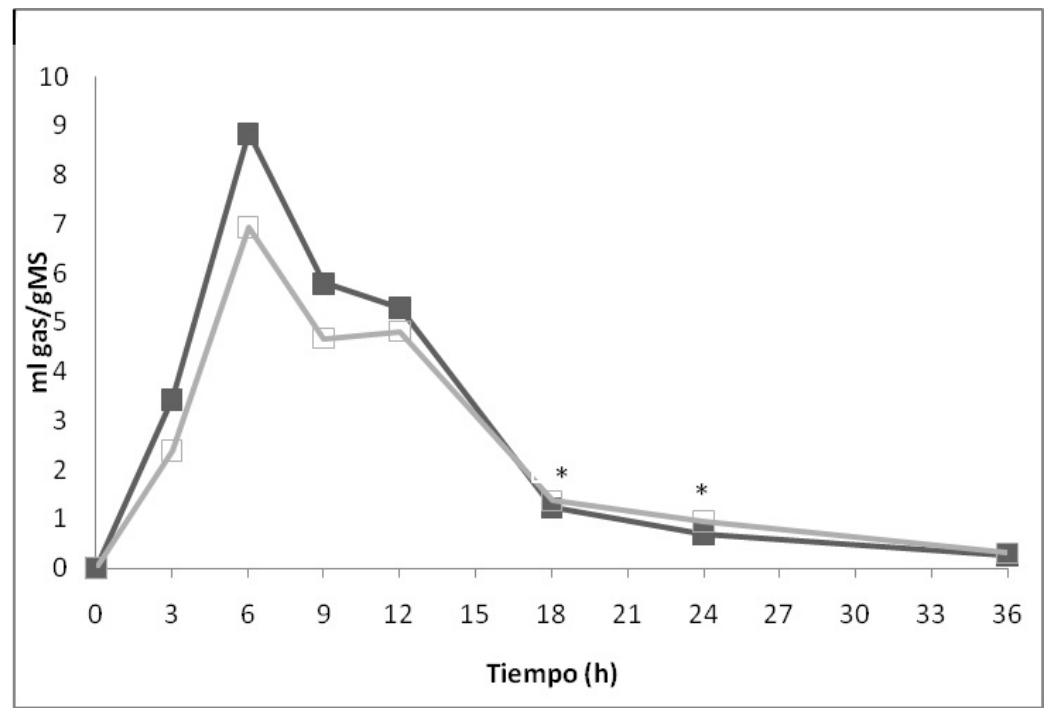

Figura 2. Producción de gas in vitro $\left(\mathrm{ml}\right.$ gas $\left.\mathrm{g}^{-} 1 \mathrm{MS}\right)$ por hora del contenido ileal inoculado con $\mathrm{Cr}(\boldsymbol{\square})$ y $\sin \mathrm{Cr}(\square)(*, \mathrm{p}<0.01)$.

Tabla 2. Contenido de cromo $\left(\mathrm{mg} \mathrm{kg}^{-1}\right), \mathrm{N}$ total, $\mathrm{N}-\mathrm{NH} 3, \mathrm{~N}$ aminoacidico y FND en la dieta, contenido ileal y cecal de cerdos Pelón Mexicano (CPM) y Cuino Mexicano (CCM) suplementados con y sin cromo.

\begin{tabular}{|c|c|c|c|c|c|c|c|c|}
\hline \multirow[t]{2}{*}{ Item } & \multicolumn{2}{|c|}{ Genotipo } & \multicolumn{2}{|c|}{ Sustrato } & \multicolumn{4}{|c|}{$\mathrm{p}<$} \\
\hline & CPM & CCM & $\mathrm{Cr}$ & $\sin C r$ & EEM & Genotipo & Sustrato & $\mathrm{G} * \mathrm{~S}$ \\
\hline \multicolumn{9}{|l|}{ Cromo } \\
\hline Dieta & 0.529 & 0.529 & 0.654 & 0.404 & & & & \\
\hline Ileal & 2.25 & 2.11 & 2.17 & 2.28 & 0.08 & 0.373 & 0.949 & 0.092 \\
\hline $\begin{array}{l}\text { Cecal } \\
\text { FND }\end{array}$ & 28.12 & 25.01 & $42.50^{a}$ & $10.63^{b}$ & 4.96 & 0.752 & 0.010 & 0.786 \\
\hline Dieta & 15.9 & 15.9 & 15.9 & 15.9 & & & & \\
\hline Cecal & 44.45 & 41.79 & 44.11 & 42.12 & 1.142 & 0.138 & 0.253 & 0.766 \\
\hline $\begin{array}{l}\mathrm{N} \text { dieta } \\
\mathrm{N} \text { ileal }\end{array}$ & 2.49 & 2.49 & 2.49 & 2.49 & & & & \\
\hline $\mathrm{N}$ total & $1.92^{a}$ & $2.63^{b}$ & 2.13 & 2.42 & 0.143 & 0.004 & 0.178 & 0.535 \\
\hline $\mathrm{N}-\mathrm{NH}_{3}$ & $0.32^{a}$ & $0.73^{b}$ & 0.47 & 0.59 & 0.094 & 0.009 & 0.385 & 0.854 \\
\hline $\begin{array}{l}\mathrm{N} \text { aa } \\
\mathrm{N} \text { Cecal }\end{array}$ & 1.60 & 1.89 & 1.66 & 1.83 & 0.139 & 0.163 & 0.412 & 0.447 \\
\hline $\mathrm{N}$ total & 3.16 & 3.29 & 3.22 & 3.23 & 0.070 & 0.254 & 0.909 & 0.615 \\
\hline $\mathrm{NNH}_{3}$ & 0.75 & 0.66 & 0.79 & 0.62 & 0.155 & 0.704 & 0.445 & 0.179 \\
\hline $\mathrm{N}$ aa & 2.41 & 2.62 & 2.42 & 2.61 & 0.162 & 0.391 & 0.436 & 0.141 \\
\hline
\end{tabular}

${ }^{a b}$ Distinta letra en la misma hilera son estadísticamente diferentes $(p<0.05)$. EEM: Error estándar de la media.

cide con los resultados del presente estudio, ya que la mayor producción de gas ocurrió a las $6 \mathrm{~h}$ (Figuras 1 y 2), lo que representa más de $40 \%$ de la FND en el contenido cecal (Tabla 2) como sustrato potencial para la fermentación microbiana.

El grado de fermentación depende de la fuente de fibra (Högberg et al. 2004, Domínguez
Vara et al. 2010); así como por la presencia de nitrógeno, minerales y vitaminas, esenciales para una nutrición de la población microbiana que reside en el intestino grueso. En los cerdos, los cambios en la micro flora cecal están influenciados por el contenido de fibra de la dieta (Högberg et al. 2004, Suarez-Beloch et al. 2013) y también determinan 
el patrón de fermentación del sustrato que llega al ciego, lo que puede estar influenciado por el tipo de micro flora presente en el ciego; en el presente estudio ambas dietas tuvieron el mismo contenido de FND, se ha documentado que ambos genotipos de cerdos poseen mayor eficiencia en la fermentación cecal con respecto a genotipos de cerdos comerciales (Suarez-Beloch et al. 2013). Por otra parte, el tipo de fermentación cecal en el cerdo varía en función del tipo de carbohidrato presente como sustrato (Bindelle et al. 2007, Domínguez-Vara et al. 2010). El intestino grueso es la sección más colonizada del tracto gastrointestinal del cerdo, hay evidencia de que la interacción bacteriana es uno de los factores más importantes que influyen en la microflora de los animales monogástricos (SuarezBeloch et al. 2013). Se sabe que de 30 a $60 \%$ del contenido gastrointestinal total de los cerdos se encuentra en el intestino grueso y permanece de 20 a $38 \mathrm{~h}$, en comparación el contenido digestivo del cerdo permanece $6 \mathrm{~h}$ en el estómago y de 2 a $6 \mathrm{~h}$ en el intestino delgado (Low y Zebrowska 1989).

En el cerdo, la absorción de nitrógeno está influenciada por el consumo de Cr orgánico en la dieta (Kornegay et al. 1997), y se relaciona con la digestión de la materia seca. Los valores de la Tabla 2 muestran que hubo una mayor concentración de Ntotal en el contenido cecal, con respecto al ileal, en ambos genotipos de cerdos, esto es evidencia de un importante crecimiento microbiano a nivel del ciego, por lo tanto del proceso de fermentación cecal, al utilizar el sustrato ileal como fuente de nutrientes como lo indican Canibe et al. (2003) para los carbohidratos de fácil fermentación. De forma similar, los valores de nitrógeno total, nitrógeno amoniacal y nitrógeno de aminoácidos, fueron mayores en el contenido cecal en ambas dietas, con y $\sin \mathrm{Cr}$, lo que demuestra un desarrollo microbiano importante a nivel cecal, así como la posible desaparición de otros componentes de la dieta durante la fermentación, como pueden ser los carbohidratos fácilmente fermentables (Canibe et al. 2003). Lo anterior, ocurre cuando se expresa en $\mathrm{mg}$ por $100 \mathrm{~g} \mathrm{MS}$, del incremento relativo de los componentes fermentados en el ciego (Tabla 2).
El intestino grueso es un órgano en el que los nutrientes no digeridos en el intestino delgado son aprovechados mediante la fermentación bacteriana, la combinación de una densidad optima y la complejidad de la microbiota, pueden contribuir para lograr un mayor beneficio/costo (Gaskins 1997), con mayor producción de $\mathrm{N}-\mathrm{NH}_{3}$ a nivel cecal. Las bacterias cecales proporcionan nutrientes al huésped, tales como ácidos grasos volátiles, vitamina $\mathrm{K}$, vitaminas del complejo $\mathrm{B}$, y aminoácidos (Savage 1986). Sin embargo, el interés de investigar los beneficios que proporciona la microbiota intestinal en la nutrición del cerdo se ha centrado en el tipo de microorganismos y su distribución espacial en el ciego y colon; así como en la producción de ácidos grasos volátiles, y los gases hidrógeno y metano, en respuesta a diversas fuentes de energía dietaria (Jensen y Jørgensen 1994). Como se muestra en el presente estudio, no hay un efecto del suministro del $\mathrm{Cr}$ en el crecimiento microbiano, la fermentación cecal, el contenido de $\mathrm{N}$-total, $\mathrm{N}$ $\mathrm{NH}_{3}$ y $\mathrm{N}$-aa en el ciego. Aunque la fibra dietética y el almidón, fisiológicamente resistente, no son fácilmente digeridos por las enzimas del huésped (Englyst 1989), son los sustratos primarios para la digestión y la fermentación microbiana en el intestino grueso (Domínguez-Vara et al. 2010), asi mismo todas las sustancias orgánicas en el intestino grueso, son posibles sustratos potenciales para la fermentación microbiana cecal (Yen 2001).

\section{CONCLUSIONES}

A partir de las $18 \mathrm{~h}$ de incubación, el CPM tuvo mayor fermentación cecal que el CCM; por lo que el CCM tuvo mayor contenido de $\mathrm{N}$-Total y $\mathrm{N}$ $\mathrm{NH}_{3}$ en el íleon. El cromo suministrado en la dieta no afectó el contenido de nitrógeno en el íleon y ciego, ni la fermentación cecal microbiana de los cerdos. Se requieren estudios más precisos para dilucidar los efectos del $\mathrm{Cr}$ dietario en los procesos de fermentación microbiana y caracterizar mejor los mecanismos de fermentación microbiana para cuantificar los aportes de nutrientes en el intestino grueso de este tipo de cerdos. 


\section{LITERATURA CITADA}

Allan JE (1971) The preparation of agricultural samples for analysis by atomic absorption spectroscopy. Varian Techtron. California. 15p.

Amoikon EK, Fernandez JM, Southern LL, Thompson DL, Ward TL, Olcott BM (1995) Effect of chromium tripicolinate on growth, glucose tolerance, insulin sensitivity, plasma metabolites, and growth hormone in pigs. Journal of Animal Science 73: 1123-1130.

AOAC (1990) Official Methods of Analysis of the Association of Official Analytical Chemists. 15th ed. The Association: Arlington, VA, USA. 148p.

Arvizu RR, Domínguez IA, Rubio MS, Borquez JL, Pinos-Rodríguez JM, González M (2011) Effects of genotype, level of supplementation, and organic chromium on growth performance, carcass, and meat traits grazing lambs. Journal of Meat Science 88: 404-408.

Bindelle J, Buldgen A, Michaux D, Wavreille J, Destain JP, Leterme P (2007) Influence of purified dietary fiber on bacterial protein synthesis in the large intestine of pigs, as measured by the gas production technique. Livestock Science 109: 232-235.

Canibe N, Peisker M, Jensen Sk, Jensen BB (2003) Effect of fermentation of feed on lysine degradation. In: Ball RO (Ed.). Proceedings of the ninth international symposium on digestive physiology of the pig, vol. 2. University of Alberta, Edmonton, Banff, Alta, Canada. pp.244-246.

Cummings JH (1983) Fermentation in the human large intestine: evidence and implications for health. The Lancet 231: 1206-1209.

De Schrijver R, Vanhoof K, Vande Ginste J (1999) Effect of enzyme resistant starch on large bowel fermentation in rats and pigs. Nutrition Research 19: 927-936.

Domínguez-Vara IA, Lara-Fuentes LA, García-Montes de OCA, Romero-Bernal J, Pescador-Salas N, González-Ronquillo M (2010) Influence dietary fiber upon in vitro microbial cecal fermentation in Mexican hairless and Mexican cuino pigs. Tropical and Subtropical Agroecosystems 12: 531-537.

Durmic Z, Pethick DW, Mullan BP, Schulze H, Accioly JM, Hampson DJ (2000) Extrusion of wheat or sorghum and/or addition of exogenous enzymes to pig diets influences the large intestinal microbiota but does not prevent development of swine dysentery following experimental challenge. Journal of Applied Microbiology 89: 678-686.

Englyst H (1989) Classification and measurement of plant polysaccharides. Animal Feed Science and Technology 23: $27-42$.

FAO (2007) Global plan of action for animal genetic resources and the Interlaken declaration. Commission on Genetic Resources for Food and Agriculture (FAO). Roma. 37p.

Firkins JL, Weiss WP, Piwonka EJ (1992) Quantification of intraruminal recycling of microbial nitrogen using nitrogen15. Journal of Animal Science 70: 3223-3233.

Fondevila M, Morales J, Pérez JF, Barrios-Urdaneta A, Baucells MD (2002) Microbial cecal fermentation in Iberic or Landrace pigs given acorn/sorghum or maize diets estimated in vitro using the gas production technique. Animal Feed Science and Technology 102: 93-107.

France J, Dhanoa MS, Theodorou MK, Lister SJ, Davies DR, Isac D (1993) A model to interpret gas accumulation profiles associated with in vitro degradation of ruminant feeds. Journal of Theoretical Biology 163: 99-111.

García-Castillo RF, Gutiérrez-Bañuelos H, Mellado-Bosque M, Morones-Reza M (2004) Cromo L-metionina en dietas basadas en sorgo y soya en cerdas primerizas. Revista Agraria-Nueva Época 1: 6-11.

Gaskins HR (1997) Immunological aspects of host/microbiota interactions at the intestinal epithelium. In: Mackie RI, White BA, Isaacson RE (eds.) Gastrointestinal microbiology. Chapman and Hall. New York. pp.537-587.

Grafit (1992) Version 13. Data Analysis and Graphics Program. Erithacus Software Ltd.

Hume ID (1997) Fermentation in the hindgut of mammals. In: Mackie RI, White BA (eds.). Gastrointestinal Microbiology Chapman and Hall New York. pp: 84-115. 
Högberg A, Lindberg JE, Leser T, Wallgren P (2004) Influence of cereal non starch polysaccharides on lleo-caecal and rectal microbial populations in Growing pigs. Acta Veterinaria Scandinavica 45: 87-98.

Jensen BB, Jørgensen H (1994) Effects of dietary fiber on microbial activity and microbial gas production in various regions of the gastrointestinal tract of pigs. Applied and Environmental Microbiology 60: 1897-1904.

Jezierny D, Steinga H, Drochner W (2007) In vitro gas formation and fermentation parameters using different substrates and pig fecal inocula affected by bile extract. Livestock Science 109: 145-148.

Kitchalong L, Fernández JM, Bunting LD, Southern LL, Bidner TD (1995) Influence of chromium tripicolinate on glucose metabolism and nutrient partitioning in growing lambs. Journal of Animal Science 73: 2694-2705.

Kornegay ET, Wang Z, Wood CM, Lindemann MD (1997) Supplemental chromium picolinate influences nitrogen balance, dry matter digestibility, and carcass traits in growing- finishing pigs. Journal of Animal Science 75: 1319-1323.

Lemus FC, Alonso MR, Alonso-Spilsbury M, Ramírez NR (2003) Morphologic characteristics in Mexican native pigs. Archivos de Zootecnia 52: 105-108.

Lindemann MD, Wood CM, Harper AF, Kornegay ET, Anderson RA (1995) Dietary chromium picolinate additions improve gain: Feed and carcass characteristics in growing finishing pigs and increase litter size in reproducing sows. Journal of Animal Science 73: 457-465.

Low AG, Zebrowska T (1989) Digestion in pigs. In: Bock HD, Eggum BO, Low AG, Simon O, Zebrowska T (eds.) Protein in metabolism in farm animals. Evaluation digestion, absorption and metabolism. Oxford University Press Oxford. pp. 53-121.

Mertz W (1993) Chromium in human nutrition: A review. Journal of Nutrition 123: 626-633.

Regmi PR, Mezler-Zebeli BU, Gänzle MG, Van Kempen TATG, Ziljstra RT (2011) Starch with high amylose content and low in vitro digestibility increases intestinal nutrient flow and microbial fermentation and selectively promotes Bifidobacteria in pigs. Journal of Nutrition 141: 1273-1280.

SAS (1999) Static Analysis System. Institute INC., Cary. NC USA. Release 9.1. pp.3891-4012.

Savage DC (1986) Gastrointestinal microflora in mammalian nutrition. Annual Reviews in Nutrition 6: 155-178.

Sierra-Vásquez AC (2000) Conservación genética del cerdo Pelón Mexicano en Yucatán y su integración a un sistema de producción sostenible: Primera aproximación. Archivos de Zootecnia 49: 415-421.

Steel RGD, Torrie JH, Dickey DA (1997) Principles and procedures of statistics: a biometrical approach. 3rd ed. McGraw-Hill. USA. 666p.

Suarez-Belloch J, Doti S, Rodríguez-Romero N, Guada JA, Fondevila M, Latorre M (2013) Hindgut fermentation in pigs induced by diets with different sources or starch. Spanish Journal of Agricultural Research 11: 780-789.

Theodorou MK, Williams BA, Dhanoa MS, McAllan AB, France J (1994) A simple gas production method using a pressure transducer to determine the fermentation kinetics of ruminate. Animal Feed Science and Technology 48: 185-197.

Van Soest PJ, Robertson JB, Lewis BA (1991) Methods for dietary fiber, neutral detergent fiber, and nonstarch polysaccharides in relation to animal nutrition. Journal of Dairy Science 74: 3583-3597.

Williams BA, Verstegen MWA, Tamminga S (2001) Fermentation in the large intestine of single stomached animals and its relationships to animal health. Nutrition Research Reviews 14: 207-227.

Yen JT (2001) Digestive system. In: Pond WG, Mersmeann HJ (eds). Biology of the domestic pig. Comstock publishing associates USA. pp: 399- 401.

Zervas S, Zijlstra RT (2002) Effects of dietary protein and fermentable fiber on nitrogen excretion patterns and plasma urea in grower pigs. Journal Animal Science 80: 3238-3246. 
